\title{
On Born-Infeld Gravity in Weitzenböck spacetime
}

\author{
Rafael Ferraro 1,2 , 田 and Franco Fiorini ${ }^{1,0}$ \\ ${ }^{1}$ Instituto de Astronomía y Fúsica del Espacio, Casilla de Correo 67, Sucursal 28, 1428 Buenos Aires, Argentina \\ ${ }^{2}$ Departamento de Física, Facultad de Ciencias Exactas y Naturales, \\ Universidad de Buenos Aires, Ciudad Universitaria, Pabellón I, 1428 Buenos Aires, Argentina
}

\begin{abstract}
Using the Teleparallel Equivalent of General Relativity formulated in Weitzenböck spacetime, we thoroughly explore a kind of Born-Infeld regular gravity leading to second order field equations for the vielbein components. We explicitly solve the equations of motion for two examples: the extended BTZ black hole, which results to exist even if the cosmological constant is positive, and a cosmological model with matter, where the scale factor results to be well behaved, giving so a singularity-free solution.
\end{abstract}

\section{INTRODUCTION: ULTRAVIOLET CORRECTIONS TO GR}

In the last decade a wide variety of modified theories of gravity has been studied with the aim of solving or smoothing some puzzling features of conventional gravity and cosmology such as singularities, particle horizons, acceleration of the universe expansion, etc. Many of these modified theories of gravity consist in the mere deformation of the current theory. In this case, one starts from a known Lagrangian $\mathcal{L}=e L$, where $L$ is invariant and $e$ is a density under general coordinate changes, and then the theory is deformed by replacing the Lagrangian by $\mathcal{L}_{D}=e f(L)$. It is expected that a suitable choice of the function $f$ will heal the unwanted features of the original theory. To explain the method, let us consider an invariant Lagrangian $L=L\left(\phi^{a}, \phi_{, \mu}^{a}, \phi_{, \mu \nu}^{a}, \ldots, x^{\mu}\right)$ and a density $e$ that does not depend on the derivatives of the fields $\phi^{a}: e=e\left(\phi^{a}, x^{\mu}\right)$ (this is because $\phi^{a}$ will later become a field describing the geometry, and so the density $e$ will be the square root of the determinant of the metric). Thus the Euler-Lagrange equations for the deformed Lagrangian $\mathcal{L}_{D}=\operatorname{e} f(L)$ are

$$
\begin{aligned}
0= & \ldots-\partial_{\mu} \partial_{\nu}\left(\frac{\partial \mathcal{L}_{D}}{\partial \phi_{, \mu \nu}^{a}}\right)+\partial_{\mu}\left(\frac{\partial \mathcal{L}_{D}}{\partial \phi_{, \mu}^{a}}\right)-\frac{\partial \mathcal{L}_{D}}{\partial \phi^{a}}= \\
& \ldots-\partial_{\mu} \partial_{\nu}\left(f^{\prime}(L) \frac{\partial \mathcal{L}}{\partial \phi_{, \mu \nu}^{a}}\right)+\partial_{\mu}\left(f^{\prime}(L) \frac{\partial \mathcal{L}}{\partial \phi_{, \mu}^{a}}\right) \\
& -f^{\prime}(L) \frac{\partial \mathcal{L}}{\partial \phi^{a}}+\left(L f^{\prime}(L)-f(L)\right) \frac{\partial e}{\partial \phi^{a}}
\end{aligned}
$$

If the deformed Lagrangian is intended to modify only the strong field (large $L$ ) regime, then $f$ should satisfy

$$
f(L) \simeq L+O\left(L^{2}\right)
$$

i.e.,

$$
f(0)=0, \quad f^{\prime}(0)=1 .
$$

*Electronic address: ferraro@iafe.uba.ar Member of Carrera del Investigador Científico (CONICET, Argentina)

${ }^{\dagger}$ Electronic address: franco@iafe.uba.ar
In general, equations (11) will have solutions differing from those coming from the original Lagrangian $\mathcal{L}=e L$. However, it should be noted that not all the solutions of the original theory get deformed by this procedure. In fact, let us consider solutions of the original theory such that $L=0$. In this case, by substituting $L=0$ in (1) and using (3) it results that the last term vanishes. Moreover, since $f^{\prime}(0)=1$, then those solutions of the original theory having $L=0$ also solve the Euler-Lagrange equations for the deformed Lagrangian $\mathcal{L}_{D}$. In particular, the invariant $L$ for general relativity (GR) is the curvature scalar $R$ associated with the Levi-Civita connection, which is null for all the (vacuum) solutions. Thus general relativity is a quite rigid theory, because its vacuum solutions remain as solutions for the (vacuum) field equations of deformed theories $\mathcal{L}_{D} \propto \sqrt{-g} f(R)$, with $f$ satisfying conditions (3). This is a rather singular feature which is not shared by other field theories. For instance, in Maxwell electromagnetism it is $L \propto E^{2}-B^{2}$, and only some vacuum solutions -mainly plane waves- make null the Maxwell Lagrangian.

Contrasting with other theories, general relativity Lagrangian $L \propto R$ contains second derivatives of the metric. In spite of this feature, Einstein equations result to be second order because the fourth order terms in EulerLagrange equations cancel out (in other words, second derivatives in $L$ appear just contributing to a divergence term in the action). This property is lost in the deformed theory $\mathcal{L}_{D} \propto \sqrt{-g} f(R)$, whose dynamical equations become fourth order, as it follows from Eq. (1). This undesirable fact is usually relieved by splitting the metric in a new metric tensor times a conformal factor depending on a scalar field; the scalar field becomes a constant when the $\left(f^{\prime}=1\right)$ general relativity case is retrieved. This procedure allows to reformulate a $f(R)$ theory as a Brans-Dicke-like scalar-tensor theory of gravity having $\omega=0$ (metric formalism [1, 2]) or $\omega=-3 / 2$ (Palatini formalism [3, 4, 5]); thus the new metric results to be governed by second order equations and the extra degrees of freedom are placed in a scalar field fulfilling a second order equation too. However, the scalar-tensor reformulation of $f(R)$ theories results in violations of the weak equivalence principle, since matter and gravity would couple not only through the (new) metric but 
also through the scalar field [6, 7]. Incidentally, we mention that not all the $f(R)$ 's appearing in the literature fulfill the conditions (3); see, for instance, the $f(R)$ used to build the spherically symmetric vacuum solution in Ref. [8], or the one proposed in Ref. [4, 9] to explain the acceleration of the universe as an effect of modified gravity at the low curvature regime (which, if regarded as a Brans-Dicke-like theory, can be dismissed on the basis of well established post-Newtonian constraints 2, 5, 10]; the Newtonian limit in Palatini formalism is not retrieved either [11]).

The problems inherent in the formulation of a $f(R)$ theory can be avoided by starting from an alternative theory of gravity whose Lagrangian only contains first derivatives of the dynamical variables. In a recent article [12] we have proposed to deform the teleparallel equivalent of general relativity TEGR 13]. As currently formulated, TEGR is an alternative formulation of general relativity. Although the dynamical object of the theory is not the metric but the vielbein $e_{\mu}^{a}(x)$, the teleparallel action is invariant under local Lorentz transformations $\Lambda_{a}^{a^{\prime}}(x)$ of the vielbein,

$$
e_{\mu}^{a}(x) \rightarrow e_{\mu}^{a^{\prime}}(x)=\Lambda_{a}^{a^{\prime}}(x) e_{\mu}^{a}(x),
$$

which do not change the metric

$$
g_{\mu \nu}(x)=\eta_{a b} e_{\mu}^{a}(x) e_{\nu}^{b}(x),
$$

where $\eta_{a b}=\operatorname{diag}(1,-1,-1, .$.$) . Since TEGR action is$ not sensitive to some of the degrees of freedom of the vielbein, the theory can be driven to be equivalent to general relativity for the metric (5) [14, 15]. Teleparallel Lagrangian is built from the torsion associated with the Weitzenböck connection [16]

$$
\stackrel{W}{\Gamma}_{\mu \nu}^{\lambda}=e_{a}^{\lambda} \partial_{\nu} e_{\mu}^{a}=-e_{\mu}^{a} \partial_{\nu} e_{a}^{\lambda},
$$

where $e_{a}^{\lambda}$ stands for the vielbein inverse matrix:

$$
e_{a}^{\mu} e_{\mu}^{b}=\delta_{a}^{b}, \quad e_{a}^{\mu} e_{\nu}^{a}=\delta_{\nu}^{\mu} .
$$

Weitzenböck connection has zero Riemann curvature $\stackrel{W}{R}$, but non-null torsion:

$$
T_{\mu \nu}^{\lambda}=\stackrel{W}{\Gamma}_{\nu \mu}^{\lambda}-\stackrel{W}{\Gamma}_{\mu \nu}^{\lambda}=e_{a}^{\lambda}\left(\partial_{\mu} e_{\nu}^{a}-\partial_{\nu} e_{\mu}^{a}\right) .
$$

The structure of the torsion tensor resembles the one of the electromagnetic field tensor. Moreover, like Maxwell, teleparallel Lagrangian density is quadratic in this tensor. In fact, TEGR Lagrangian with cosmological constant $\Lambda$ is 17

$$
\mathcal{L}_{\mathbf{T}}\left[e_{\mu}^{a}(x)\right]=\frac{e}{16 \pi G}(\mathbb{S} \cdot \mathbb{T}-2 \Lambda),
$$

where $e$ is the determinant of matrix $e_{\mu}^{a}$ (which is equal to $\sqrt{-g}), \mathbb{S} \cdot \mathbb{T} \doteq S_{\rho}{ }^{\mu \nu} T^{\rho}{ }_{\mu \nu}$, and $S_{\rho}{ }^{\mu \nu}$ is defined as

$$
\begin{aligned}
S_{\rho}{ }^{\mu \nu}= & -\frac{1}{4}\left(T_{\rho}^{\mu \nu}-T_{\rho}^{\nu \mu}-T_{\rho}{ }^{\mu \nu}\right) \\
& +\frac{1}{2} \delta_{\rho}^{\mu} T_{\theta}^{\theta \nu}-\frac{1}{2} \delta_{\rho}^{\nu} T_{\theta}^{\theta \mu} .
\end{aligned}
$$

While Einstein-Hilbert Lagrangian depends on second derivatives of the metric, teleparallel Lagrangian is built just with first derivatives of the vielbein, which makes more attractive the study of its deformation, in the sense that the field equations of the deformed theory will remain being second order equations. The Euler-Lagrange equations for the Lagrangian $\mathcal{L}_{\mathbf{T}}+\mathcal{L}_{\text {matter }}$ are

$$
\begin{aligned}
\partial_{\sigma}\left(e e_{a}^{\lambda} S_{\lambda}{ }^{\nu \sigma}\right)-e e_{a}^{\lambda} S_{\eta}{ }^{\mu \nu} T^{\eta}{ }_{\mu \lambda} & +\frac{1}{4} e e_{a}^{\nu}(\mathbb{S} \cdot \mathbb{T}-2 \Lambda) \\
& =4 \pi G e e_{a}^{\lambda} T_{\lambda}{ }^{\nu},(11)
\end{aligned}
$$

where $T_{\lambda}{ }^{\nu}$ is the matter energy-momentum tensor. By contracting Eq. (11) with the inverse vielbein $e_{\nu}^{a}$ one obtains for vacuum solutions

$$
4 e^{-1} e_{\nu}^{a} \partial_{\sigma}\left(e e_{a}^{\lambda} S_{\lambda}^{\nu \sigma}\right)+(n-4) \mathbb{S} \cdot \mathbb{T}=2 n \Lambda,
$$

being $n$ the spacetime dimension. In contrast to general relativity, where Einstein equations compels $R$ to vanish in vacuum (or to be a constant when the cosmological constant is included), Eq. (12) does not compel the invariant $\mathbb{S} \cdot \mathbb{T}$ to be null nor constant for vacuum solutions, which raises the hope that a deformed teleparallelism could be useful to smooth singularities of vacuum general relativity solutions.

\section{BORN-INFELD GRAVITY}

Born-Infeld (BI) electrodynamics 18] has experienced a renewed interest in the last years due to its close connection with string theory, particularly because its capability to describe the electromagnetic fields of D-branes [19], [20]. Inspired in these fruitful properties, together with the ability of BI program concerning the cure of singularities, we shall study a teleparallel theory of gravity deformed à la Born-Infeld. In a rather different approach, this subject has received some attention in the past [21, 22, 23, 24, 25, 26], where several deformations à la Born-Infeld combining higher order invariants constructed with the curvature in a Riemannian context were tried. All these constructions, however, lead to troublesome four order field equations for the metric. As a matter of fact, explicit solutions in four dimensions within these frameworks were never found [33]. In a different direction, BI actions were explored more recently in Ref. [27, 28] using the Palatini formalism, where metric and connection are taken as independent entities. In turn, following the lines of [12], we will work with the Lagrangian

$$
\mathcal{L}_{\mathrm{BI}}\left[e_{\mu}^{a}(x)\right]=\frac{\lambda e}{16 \pi G}\left[\sqrt{1+\frac{2(\mathbb{S} \cdot \mathbb{T}-2 \Lambda)}{\lambda}}-1\right],
$$

where $\lambda$ is a constant that controls the scale at which the deformed solutions depart from the original ones: Lagrangian (13) tends to (9) when $\lambda \rightarrow \infty$. According to 
Eq. (1), the Euler-Lagrange equations become

$$
\begin{aligned}
& \partial_{\sigma}\left[\left(1+2 \lambda^{-1}(\mathbb{S} \cdot \mathbb{T}-2 \Lambda)\right)^{-1 / 2} e e_{a}^{\lambda} S_{\lambda}{ }^{\nu \sigma}\right] \\
& -\left(1+2 \lambda^{-1}(\mathbb{S} \cdot \mathbb{T}-2 \Lambda)\right)^{-1 / 2} e e_{a}^{\lambda} S_{\eta}^{\mu \nu} T^{\eta}{ }_{\mu \lambda} \\
& +\frac{\lambda}{4} e e_{a}^{\nu}\left[\left(1+2 \lambda^{-1}(\mathbb{S} \cdot \mathbb{T}-2 \Lambda)\right)^{1 / 2}-1\right] \\
= & 4 \pi G e e_{a}^{\lambda} T_{\lambda}^{\nu} .
\end{aligned}
$$

In order to explore the aptitude of deformed teleparallelism to modify solutions of general relativity, we will try two types of examples: the BTZ black hole and a ndimensional cosmological model with matter. In the first example, both GR and teleparallel Lagrangians result to be constant for the chosen solution, thus the deformation is limited to a shift of the cosmological constant. In spite of this, teleparallelism exhibits a better aptitude to deform the solution because it allows for a BTZ solution even for positive cosmological constant. The strength of modified teleparallelism is however revealed in solutions with sources, where modified teleparallelism is able to control the growing of the Hubble parameter by avoiding that the universe reaches a singularity in a finite time.

\section{A. Extended BTZ black hole}

BTZ black hole is a vacuum solution for general relativity with negative cosmological constant $\Lambda$ in $2+1$ dimensions [29]. The spinning BTZ metric is

$$
\begin{aligned}
& d s^{2}=\left(-M-\Lambda r^{2}+\frac{J^{2}}{4 r^{2}}\right) d t^{2} \\
& -\left(-M-\Lambda r^{2}+\frac{J^{2}}{4 r^{2}}\right)^{-1} d r^{2}-r^{2}\left(-\frac{J}{2 r^{2}} d t+d \phi\right)^{2}
\end{aligned}
$$

where $M$ and $J$ are integration constants related to the mass and the angular momentum respectively. For $\Lambda=-\ell^{-2}, M>0$ and $|J| \leq M \ell$ this metric has the structure of a rotating black hole. The BTZ black hole displays event horizons (the place where the lapse function vanishes) at 30]

$$
r^{ \pm}=\ell\left[\frac{M}{2} \pm \frac{M}{2} \sqrt{1-\left(\frac{J}{M \ell}\right)^{2}}\right]^{1 / 2},
$$

and the ergosphere (the place where $g_{t t}$ vanishes) at

$$
r^{e r g}=\ell M^{1 / 2}>r^{+}>r^{-} .
$$

The extremal case $|J|=M \ell$ corresponds to $r^{+}=r^{-}=$ $r_{\text {erg }} / \sqrt{2}$. A suitable dreibein field for the metric (15) is given by

$$
\begin{aligned}
e^{0} & =\left(-M-\Lambda r^{2}+\frac{J^{2}}{4 r^{2}}\right)^{1 / 2} d t \\
e^{1} & =\left(-M-\Lambda r^{2}+\frac{J^{2}}{4 r^{2}}\right)^{-1 / 2} d r \\
e^{2} & =-\frac{J}{2 r} d t+r d \phi .
\end{aligned}
$$

This dreibein satisfies Eq. (11) for vanishing energymomentum tensor, and $\eta_{a b} e^{a} e^{b}$ reproduces the interval (15). Let us investigate how this solution is affected by a deformation of the theory. In order to understand the changes that the dreibein (18) has to undergo for becoming a solution of the deformed equations (14), let us note that the invariant $\mathbb{S} \cdot \mathbb{T}$ results to be constant for the dreibein (18): its value is $-2 \Lambda$. Although $\mathcal{L}_{\mathbf{T}}$ is not zero, a vacuum solution like (18) which renders $\mathcal{L}_{\mathrm{T}}=$ constant is very close to a vacuum solution of the deformed theory. In fact, let us modify solution (18) by replacing $\Lambda$ with a new constant $\widetilde{\Lambda}$. Then $\mathbb{S} \cdot \mathbb{T}=-2 \widetilde{\Lambda}$, so Eq. (14) turns out to be

$$
\begin{gathered}
\partial_{\sigma}\left(e e_{a}^{\lambda} S_{\lambda}{ }^{\nu \sigma}\right)-e e_{a}^{\lambda} S_{\eta}{ }^{\mu \nu} T^{\eta}{ }_{\mu \lambda} \\
+\frac{1}{4} e e_{a}^{\nu}[\mathbb{S} \cdot \mathbb{T}-2(2 \Lambda+\widetilde{\Lambda})+\lambda \\
\left.-\lambda\left(1-4 \lambda^{-1}(\Lambda+\widetilde{\Lambda})\right)^{1 / 2}\right]=0 .
\end{gathered}
$$

Since the solution we are trying solves the teleparallel vacuum equation (11) for $\Lambda=\widetilde{\Lambda}$, then it will solve Eq. (19) if $\widetilde{\Lambda}$ is chosen such that

$$
-2(2 \Lambda+\widetilde{\Lambda})+\lambda-\lambda\left(1-4 \lambda^{-1}(\Lambda+\widetilde{\Lambda})\right)^{1 / 2}=-2 \widetilde{\Lambda},
$$

i.e.,

$$
\widetilde{\Lambda}=\Lambda(1-\epsilon), \quad \epsilon=4 \Lambda / \lambda .
$$

This solution represents a black hole if the effective cosmological constant $\widetilde{\Lambda}$ is negative. Summarizing, the BTZ dreibein for the deformed gravity theory described by Lagrangian (13) is

$$
\begin{aligned}
e^{0} & =\left(-M-\Lambda(1-\epsilon) r^{2}+\frac{J^{2}}{4 r^{2}}\right)^{1 / 2} d t \\
e^{1} & =\left(-M-\Lambda(1-\epsilon) r^{2}+\frac{J^{2}}{4 r^{2}}\right)^{-1 / 2} d r \\
e^{2} & =-\frac{J}{2 r} d t+r d \phi,
\end{aligned}
$$

and the metric is

$$
\begin{array}{r}
d s^{2}=\left(-M-\Lambda(1-\epsilon) r^{2}+\frac{J^{2}}{4 r^{2}}\right) d t^{2} \\
-\left(-M-\Lambda(1-\epsilon) r^{2}+\frac{J^{2}}{4 r^{2}}\right)^{-1} d r^{2} \\
-r^{2}\left(-\frac{J}{2 r^{2}} d t+d \phi\right)^{2} .
\end{array}
$$

The dreibein (22) or the metric (23) genuinely differs from (18) and (15); these two metrics are not connected by a coordinate change because the invariant $\mathbb{S} \cdot \mathbb{T}$ is different for each one (also $R$ is different). For negative effective cosmological constant $\widetilde{\Lambda}=-\widetilde{\ell}^{-2}$ the solution is 
a rotating BTZ black hole. Thus, even for $\Lambda>0$ the BI Lagrangian (13) allows for BTZ rotating black holes; specifically, the metric (23) is a rotating black hole for $\Lambda<0$ and $\epsilon<1$, but also for $\Lambda>0$ and $\epsilon>1$. The horizons are placed at

$$
\begin{aligned}
& r_{B I}^{ \pm}=\frac{r_{B I}^{e r g}}{2}\left[1 \pm \sqrt{1+\Lambda(1-\epsilon)\left(\frac{J}{M}\right)^{2}}\right]^{1 / 2} \\
& r_{B I}^{e r g}=\sqrt{\frac{M}{-\Lambda(1-\epsilon)}} .
\end{aligned}
$$

Let us compare the lengths of the horizons for the solutions (15) and (22) corresponding to fixed values of $\Lambda, M$ and $J$. Since the horizons are circles, we will study the ratio $r_{B I}^{ \pm} / r^{ \pm}$. For $\widetilde{\Lambda}<0$, three ranges of the parameter $\epsilon$ can be distinguished in this comparison:

- Type I: $\epsilon<0(\Lambda<0, \lambda>0)$. It results $r_{B I}^{e r g} / r^{e r g}<1, r_{B I}^{+} / r^{+}<1$ and $r_{B I}^{-} / r^{-}>1$; then the horizons approach each other as a consequence of the deformation.

- Type II: $\epsilon>1(\Lambda>0)$. There is no black hole for the $\mathbf{G R}$ counterpart.

- Type III: $0<\epsilon<1(\Lambda<0, \lambda<0)$. It results $r_{B I}^{e r g} / r^{e r g}>1, r_{B I}^{+} / r^{+}>1$ and $r_{B I}^{-} / r^{-}<1$; in this case the horizons move away from each other as a consequence of the deformation. However the case $\lambda<0$ will be rejected in next section since it produces physically unacceptable solutions in cosmology.

This example shows the strategy to be followed to obtain deformed solutions when one starts from a Lagrangian having a "cosmological constant" term like the one in Eq. (9), i.e. $\mathcal{L} \propto e(L-2 \Lambda)$. If a given (vacuum) solution makes $L$ a ( $\Lambda$ depending) constant, then replace $\Lambda$ in the solution by a new constant $\widetilde{\Lambda}$ and substitute the so built solution in the modified field equation. Using that $L$ is constant, Eq. (11) becomes

$$
\begin{gathered}
\ldots-\partial_{\mu} \partial_{\nu}\left(e \frac{\partial L}{\partial \phi_{, \mu \nu}^{a}}\right)+\partial_{\mu}\left(e \frac{\partial L}{\partial \phi_{, \mu}^{a}}\right) \\
-e \frac{\partial L}{\partial \phi^{a}}+\left(L-\frac{f(L-2 \Lambda)}{f^{\prime}(L-2 \Lambda)}\right) \frac{\partial e}{\partial \phi^{a}}=0 .
\end{gathered}
$$

The proposed solution now solves the undeformed EulerLagrange equations for cosmological constant $\widetilde{\Lambda}$. Therefore $\widetilde{\Lambda}$ should be chosen in such a way that

$$
L(\widetilde{\Lambda})-\frac{f(L(\widetilde{\Lambda})-2 \Lambda)}{f^{\prime}(L(\widetilde{\Lambda})-2 \Lambda)}=2 \widetilde{\Lambda}
$$

where $L(\widetilde{\Lambda})$ is the Lagrangian evaluated on the proposed solution. This means that the deformation replaces the role of the cosmological constant in the solution for a new parameter depending also on the scale $\lambda$. Teleparallelism à la Born-Infeld (Lagrangian (13) ) uses the function $f$

$$
f_{\mathbf{B I}}(x)=\lambda \sqrt{1+\frac{2 x}{\lambda}}-\lambda
$$

so, writing Eq. (26) for the function (27), one gets Eq. (20) and the solution (21).

Einstein equations with cosmological constant imply $L=-R=2 \Lambda n /(n-2)$ for any vacuum solution in $n$ spacetime dimensions. Therefore, vacuum solutions for theories $f(-R-2 \Lambda)$ can be straightforwardly obtained from general relativity vacuum solutions by shifting $\Lambda$ to be

$$
\frac{2 n}{n-2} \widetilde{\Lambda}-\frac{f(2 \widetilde{\Lambda}[n /(n-2)]-2 \Lambda)}{f^{\prime}(2 \widetilde{\Lambda}[n /(n-2)]-2 \Lambda)}=2 \widetilde{\Lambda}
$$

Contrasting with teleparallel Eq. (12), the vacuum solutions of GR share the same value of $L(\Lambda)$. Thus, the effective cosmological constant (28) for modified GR is the same for all vacuum solutions.

Just for comparing with the Born-Infeld modified teleparallelism result (21), let us compute the modified GR solution (28) for the Born-Infeld deformation (27) in $n=3$ dimensions. The result is

$$
\widetilde{\Lambda}=\frac{\Lambda}{2}\left[1-\frac{1}{4 \epsilon}(1-\sqrt{1+8 \epsilon})\right] .
$$

Thus the Born-Infeld deformation for $\mathbf{G R}$ is well defined if $\epsilon>-1 / 8$, and the bracket in Eq. (29) is positive definite. This means that the effective cosmological constant keeps the sign of $\Lambda$. Therefore, in GR modified à la BornInfeld the BTZ black hole only exists for $\Lambda<0$, which is a result different from the one obtained for modified teleparallelism.

\section{B. Regular Cosmology}

The inability of Einstein-Hilbert Lagrangian to allow for high energy deformed solutions not only embraces the vacuum solutions, but any $\mathbf{G R}(\Lambda=0)$ solution satisfying $R=0$. This assertion remains valid even if there are sources. For instance, the Friedmann-Robertson-Walker (FRW) solution for a radiation fluid cannot be smoothly deformed, because the energy-momentum tensor is traceless and so it is $R=0$. In contrast, the teleparellel Lagrangian (9) does not vanish in this case; thus teleparallelism allows for a smooth deformation of such kind of solution [12].

Let us study the deformation (13) in the context of a spatially flat FRW geometry in the presence of a homogeneous and isotropic fluid. Then the source is represented by the stress-energy tensor 
$T^{\mu}{ }_{\nu}=\operatorname{diag}(\rho(t),-p(t),-p(t), \ldots)$ in the comoving reference frame. The teleparallel equations can be solved by considering the vielbein

$$
e_{\mu}^{a}=\operatorname{diag}(1, a(t), a(t), \ldots), \quad e=a^{n-1},
$$

leading to the metric $g_{\mu \nu}=\operatorname{diag}\left(1,-a(t)^{2},-a(t)^{2}, \ldots\right)$. In this case the only non null components of $\mathbb{S}$ and $\mathbb{T}$ are

$$
\begin{aligned}
& S_{\alpha 0 \alpha}=-S_{\alpha \alpha 0}=-\frac{1}{2}(n-2) a(t) \dot{a}(t), \\
& T_{\alpha 0 \alpha}=-T_{\alpha \alpha 0}=a(t) \dot{a}(t), \quad \alpha \neq 0 .
\end{aligned}
$$

Thus $\mathbb{S} \cdot \mathbb{T}=-(n-1)(n-2) H^{2}, H=\dot{a}(t) / a(t)$ being the Hubble parameter, which is not null nor constant whenever a source is present. The first term in the equation (14) for the indexes $a=0=\nu$ is null; then the initial value equation for the modified FRW cosmology results

$$
\frac{1-\epsilon}{\left(1-\epsilon-2(n-1)(n-2) \frac{H^{2}}{\lambda}\right)^{\frac{1}{2}}}-1=\frac{16 \pi G}{\lambda} \rho .
$$

The isotropy of the proposed solution makes equal the equations (14) for spatial indexes $a=\nu$; they are

$$
\begin{aligned}
& (1-\epsilon)\left(2(n-2) q \frac{H^{2}}{\lambda}+2 n(n-2) \frac{H^{2}}{\lambda}-1+\epsilon\right) \\
& \times\left(1-\epsilon-2(n-1)(n-2) \frac{H^{2}}{\lambda}\right)^{-\frac{3}{2}}+1=\frac{16 \pi G}{\lambda} p
\end{aligned}
$$

In the last expression $q=-a \dot{a}^{-2} \ddot{a}=-\left(1+\dot{H} H^{-2}\right)$ is the deceleration parameter. Eqs. (32), (33) lead to the energy-momentum conservation. In fact, by differentiating the initial value equation (32) with respect to the time, and combining this result with Eq. (33), one gets

$$
\begin{aligned}
& \frac{d}{d t}\left(\rho a^{n-1}\right)=-p \frac{d}{d t} a^{n-1}, \\
& \dot{\rho}+(n-1)(\rho+p) H=0 .
\end{aligned}
$$

For a barotropic fluid satisfying the state equation $p=$ $\omega(n) \rho$, the conservation law (34) leads to the behavior

$$
\rho(t)=\rho_{o}\left(\frac{a_{o}}{a(t)}\right)^{(n-1)(1+\omega)} .
$$

Equations (32)-(33) in vacuum $(\rho=p=0)$ have the solution $H= \pm H_{o}, q=-1$, for the constant $H_{o}^{2}=$ $2 \Lambda(1-\epsilon) /[(n-1)(n-2)]$. In this case the result is the de Sitter metric for the effective cosmological constant $\widetilde{\Lambda}=\Lambda(1-\epsilon)$. The similarity with the shift of the previous section comes from the fact that the invariant is $\mathbb{S} \cdot \mathbb{T}=$ $-2 \widetilde{\Lambda}$ in both cases.

In the presence of a barotropic fluid, the system (32), (35) can be rewritten by using the variable

$$
y=\ln \left[\left(\frac{a}{a_{o}}\right)^{(n-1)(1+\omega)}\right] \Rightarrow \dot{y}=(n-1)(1+\omega) H .
$$

Thus the dynamics of the spatially flat FRW universe in Born-Infeld teleparallelism is described by the equation

$$
\begin{aligned}
\frac{2(n-2)}{(n-1)(1+\omega)^{2}} \dot{y}^{2}+ & \frac{\lambda(1-\epsilon)^{2}}{\left(1+16 \pi G \rho_{o} \lambda^{-1} \exp (-y)\right)^{2}} \\
& =(1-\epsilon) \lambda,
\end{aligned}
$$

whose $\mathbf{G R}(\lambda \rightarrow \infty)$ limit is

$$
\frac{2(n-2)}{(n-1)(1+\omega)^{2}} \dot{y}^{2}-32 \pi G \rho_{o} \exp (-y)=4 \Lambda .
$$

The variable $y$ is monotone increasing with the scale factor $a(t)$. So the behavior of the scale factor can be read directly in the "energy conservation" equations (37) and (38). As known, the effective potential for a spatially flat FRW universe in GR expands forever for $\Lambda>0$ and recollapses for $\Lambda<0$. On the other hand, the Born-Infeld teleparallel potential for $\lambda>0$ is an increasing function, vanishing for $y \rightarrow-\infty(a \rightarrow 0)$ and going to $\lambda(1-\epsilon)^{2}$ for $y \rightarrow \infty$. Since the energy level in Eq. (37) is $\lambda(1-\epsilon)$, then: I) the universe recollapses if $1-\epsilon>1$ (i.e. $\Lambda<0$ ), or II) expands forever if $0<1-\epsilon<1$ (i.e. $0<\Lambda<\lambda / 4$ ). Although this behavior seems not to differ considerably from the GR one, it should be emphasized that the main difference lies in the behavior of the Hubble parameter when $y \rightarrow-\infty$ : while $H$ diverges in GR, in Born-Infeld teleparallelism $H$ goes to the constant value

$$
H^{2} \rightarrow \frac{(1-\epsilon) \lambda}{2(n-1)(n-2)}=\frac{\lambda-4 \Lambda}{2(n-1)(n-2)}
$$

For $\lambda<0$ and $\epsilon \neq 1$ the effective potential becomes an infinite well. This is an unphysical feature, since it leads $H$ to diverge for $16 \pi G \rho=|\lambda|$ (see Eq. (32)). Therefore we will only consider the case $\lambda>0$.

The dependence on time of the scale factor can be obtained from the initial value equation (32) or, equivalently, from (37). We will use the variable

$$
\mathrm{y}=\frac{\lambda}{16 \pi G \rho_{o}}\left(\frac{a(t)}{a_{o}}\right)^{(n-1)(1+\omega)} .
$$

In this way, the initial value equation takes the form

$$
\dot{\mathrm{y}}= \pm \mathcal{A} \frac{\mathrm{y}}{1+\mathrm{y}} \sqrt{1+2 \mathrm{y}+\epsilon \mathrm{y}^{2}}
$$

with $\mathcal{A}=(1+\omega) \sqrt{\frac{\lambda(1-\epsilon)(n-1)}{2(n-2)}}$ a non null constant. The solution of (41) can be obtained in a closed but implicit form by direct integration, and depends on the sign and range of the parameter $\epsilon$. Concretely, we have two types of solutions:

- Type $I: \epsilon<0(\Lambda<0)$ (the universe recollapses)

$$
\pm \mathcal{A} t \pm c=\mathcal{F}(\mathrm{y})-\frac{1}{(-\epsilon)^{1 / 2}} \arcsin \left[\frac{1+\epsilon \mathrm{y}}{\sqrt{1-\epsilon}}\right] .
$$


- Type II: $0<\epsilon<1(\Lambda>0)$ (the universe expands forever)

$$
\mathcal{A} t+c=\mathcal{F}(\mathrm{y})+\frac{1}{\sqrt{\epsilon}} \ln \left[\frac{1+\epsilon \mathrm{y}}{\sqrt{\epsilon}}+\sqrt{1+2 \mathrm{y}+\epsilon \mathrm{y}^{2}}\right],
$$

where the function $\mathcal{F}(\mathrm{y})$ is in both cases

$$
\mathcal{F}(\mathrm{y})=\ln \left[\frac{\mathrm{y}}{1+\mathrm{y}+\sqrt{1+2 \mathrm{y}+\epsilon \mathrm{y}^{2}}}\right] .
$$

In Eq. (42) the sign \pm corresponds to the expanding and the collapsing branch respectively. Both branches can be joined at $t=0$ by choosing the integration constant $c$ to equalize the right member for the maximum scale factor. According to Eq. (41) the maximum scale factor $(\dot{\mathrm{y}}=0)$ is

$$
\mathrm{y}_{\max }=\frac{1+\sqrt{1-\epsilon}}{-\epsilon}
$$

thus

$$
c=-\ln \left[1-\frac{\epsilon}{1+\sqrt{1-\epsilon}}\right]+\frac{\pi / 2}{(-\epsilon)^{1 / 2}} .
$$

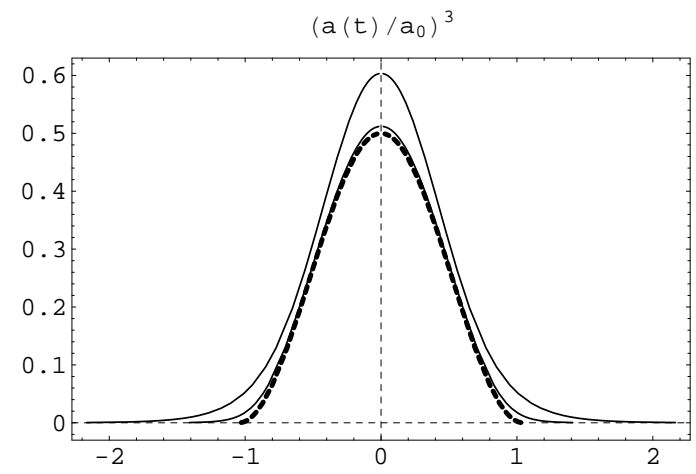

FIG. 1: Behavior of the non-dimensional cubed scale factor $\left(a(t) / a_{0}\right)^{3}$ as emerges from (42) for $\omega=1 / 2, \Lambda=-1$ in $n=3$ dimensions. The upper curve is for $\epsilon=-1$, the middle curve is for $\epsilon=-0.1$ and the dashed one corresponds to GR.

Figure 1 shows the Type I recollapsing case. The scale factor as a function of time is depicted for a radiation fluid in three dimensions, i.e. $\omega=1 / 2$ and $n=3$. Besides we set $16 \pi G \rho_{o}=1$ and $\Lambda=-1$. The upper, middle and lower (dashed) curves correspond to $\epsilon=-1(\lambda=4)$, $\epsilon=-0.1(\lambda=40)$, and GR $(\lambda \rightarrow \infty)$ respectively. Note that the GR scale factor exists only for $-1 \leq t \leq 1$, whereas it exists for all values of time in the $\mathbf{B I}$ case.

Physically more relevant, at least in four dimensions, is the Type II case where the cosmological constant is positive. In this case, the Eq. (43) says that the late time behavior $(\mathrm{y} \rightarrow \infty)$ of the scale factor is $a(t) \propto$ $\exp \left[\sqrt{\frac{2 \Lambda(1-\epsilon)}{(n-1)(n-2)}} t\right]$, while the initial stage is described by $a(t) \propto \exp \left[\sqrt{\frac{\lambda(1-\epsilon)}{2(n-1)(n-2)}} t\right]$ (see Eq. (39)). Thus, the universe evolves from an inflationary stage, driven by the (vacuum-like) energy $\lambda(1-\epsilon)$, to another exponential epoch ruled by the vacuum energy $\Lambda(1-\epsilon)$ (a similar de Sitter-de Sitter evolution was obtained in a quite different approach in Ref. [31]). Since $\epsilon$ should be very small in order that the theory does not appreciably differ from GR for most of the history of the universe (see a lower bound for $\lambda$ in Ref. [12]), one concludes that in four dimensions the scale factor evolves in time as

$$
a(t \rightarrow-\infty) \propto e^{\sqrt{\frac{\lambda}{12}} t} \rightsquigarrow a(t \rightarrow \infty) \propto e^{\sqrt{\frac{\pi}{3}} t} .
$$

Finally, the limiting case $\epsilon=1$ corresponds to the scale factor being constant, as follows from Eq. (37).

\section{CONCLUDING COMMENTS}

In spite of having different causal structures, the BTZ black hole is locally the anti-de Sitter spacetime [30], i.e. the maximally symmetric solution with negative cosmological constant. When evaluated on maximally symmetric solutions, both $R$ and $\mathbb{S} \cdot \mathbb{T}$ Lagrangians are equal to a constant that is independent of the integration constants: it only depends on $\Lambda$. So, in both theories, general relativity and teleparellelism, the deformation of these maximally symmetric solutions just amount to a shifting of the cosmological constant. The shifting is controlled by the non-dimensional parameter $\epsilon=4 \Lambda / \lambda$, where $\lambda$ is a Born-Infeld-like constant going to infinity when the deformed theory approaches the original one. The cosmological constant $\Lambda$ and the (shifted) effective cosmological constant $\widetilde{\Lambda}$ can have opposite sign in deformed teleparallelism; so the anti-de Sitter solution can solve the deformed teleparallel equations even for positive cosmological constant.

On the other hand, we have studied the deformation of non-vacuum cosmological solutions. Although the parameter $\epsilon$ takes part in Eq. (41), its presence does not alter the non-deformed result that spatially flat FriedmannRobertson-Walker non-vacuum solutions expand for $\Lambda>$ 0 and recollapse for $\Lambda<0$. Instead, the deformed theory smoothes the initial singularities, which is the effect pursued by Born-Infeld deformations. In fact, the Hubble parameter goes to a constant when the scale factor $a$ vanishes (see Eq. (39)). This value is also the maximum value to be attained by $H$ (see Eq. (32)).

The BI approach (13) generates regular solutions. In the cosmological setting this is so, not only because the scale factor is always different from zero, but because the geometrical invariants (both, in Riemann and Weitzenböck spacetimes) are bounded for all finite proper times. In fact, each invariant in Weitzenböck spacetime that is quadratic in the torsion tensor has to be proportional to $H^{2}$ in the setting (30) (see Eq. (31)). On the other hand, the Riemannian invariants for the metric $g_{\mu \nu}=\operatorname{diag}\left(1,-a(t)^{2},-a(t)^{2}, \ldots\right)$ can be cast in the polynomical form $\mathcal{P}=(H, \dot{H})$. For instance, in four dimensions, the scalar curvature is $R=6\left(2 H^{2}+\dot{H}\right)$, 
the squared Ricci scalar $R_{\mu \nu}^{2}=R^{\mu \nu} R_{\mu \nu}$ is $R_{\mu \nu}^{2}=$ $12\left(3 H^{4}+3 H^{2} \dot{H}+\dot{H}^{2}\right)$, and the Kretschmann invariant $K=R_{\beta \gamma \delta}^{\alpha} R_{\alpha}^{\beta \gamma \delta}$ reads $K=12\left(2 H^{4}+2 H^{2} \dot{H}+\dot{H}^{2}\right)$. All these invariants are well behaved due to the saturation value (39) that the Hubble parameter reaches as $a(t) \rightarrow 0$. Regarding this matter, the time derivative of Eq. (41) combined with the definition given in Eq. (40), shows that

$$
\dot{H}=-\alpha \frac{\mathrm{y}^{2}}{(1+\mathrm{y})^{3}},
$$

where $\alpha=\lambda(1+\omega)(1-\epsilon)^{2} / 2(n-2)$ is a non null constant. By setting $n=4, \omega=1 / 3$ and $\epsilon=0$, one finds the following expressions for the invariants

$$
\begin{aligned}
R & =\lambda\left[\frac{1+3 \mathrm{y}}{(1+\mathrm{y})^{3}}\right] \\
R_{\mu \nu}^{2} & =\frac{\lambda^{2}}{12}\left[\frac{3+18 \mathrm{y}+27 \mathrm{y}^{2}+4 \mathrm{y}^{4}}{(1+\mathrm{y})^{6}}\right], \\
K & =\frac{\lambda^{2}}{6}\left[\frac{1+6 \mathrm{y}+9 \mathrm{y}^{2}+4 \mathrm{y}^{4}}{(1+\mathrm{y})^{6}}\right] .
\end{aligned}
$$

This means that the BI parameter $\lambda$ not only bounds the dynamics of $H(t)$ and characterizes the minimum density for having inflation [12], but also establishes a maximum attainable curvature.

One could wonder if the BI framework here considered can be viewed as a particular case of a more general determinantal Born-Infeld action for gravity. Indeed, following in a closer way the BI spirit, one could try the n-dimensional determinantal action

$$
\mathcal{I}_{B I G}=\lambda \int d^{n} x\left[\sqrt{\operatorname{det}\left(g_{\mu \nu}+\lambda^{-1} F_{\mu \nu}\right)}-\sqrt{\operatorname{det}\left(g_{\mu \nu}\right)}\right]
$$

where $F_{\mu \nu}$ is quadratic in the Weitzenböck torsion: $F_{\mu \nu}=A S_{\mu \lambda \rho} T_{\nu}^{\lambda \rho}+B S_{\lambda \mu \rho} T_{\nu}^{\lambda}{ }^{\rho}, A$ and $B$ being nondimensional constants. Such a combination ensures the correct GR limit since both constituents of $F_{\mu \nu}$ have trace $\mathbb{S} \cdot \mathbb{T}$. Besides, the dynamical equations coming from (50) will be still of second order in the vielbein derivatives. For the choice $2 A+B=0$, the action (50) reproduces the solutions considered in the last section, though the equivalence with the scheme (13) for other solutions is not clear yet 32]. The complete characterization of the theory (50) for the whole parameter space $(A, B)$ will be matter of future developments.

\section{Acknowledgments}

We would like to thank G. Giribet for the constant encouragement afforded during this work. This research was supported by CONICET.
[1] P. Teyssandier and Ph. Tourrenc, J. Math. Phys. 24 (1983) 2793; B. Whitt, Phys. Lett. B145 (1984) 176; J.D. Barrow and S. Cotsakis, Phys. Lett. B214 (1988) 515; K. Maeda, Phys. Rev. D39 (1989) 3159.

[2] T. Chiba, Phys. Lett. B575 (2003) 1.

[3] V.H. Hamity and D.E. Barraco, Gen. Rel. Grav. 25 (1993) 461.

[4] D.N. Vollick, Phys. Rev. D68 (2003) 063510.

[5] G.J. Olmo, Phys. Rev. D72 (2005) 083505.

[6] C.H. Brans, The roots of scalar-tensor theory: an approximate history arXiv:gr-qc/0506063.

[7] G.J. Olmo, Phys. Rev. Lett. 98 (2007) 061101.

[8] T. Clifton, Class. Quant. Grav. 23 (2006) 7445.

[9] S.M. Carroll, V. Duvvuri, M. Trodden and M.S. Turner, Phys. Rev. D70 (2004) 043528.

[10] G.J. Olmo, Phys. Rev. Lett. 95 (2005) 261102.

[11] A.E. Domínguez and D.E. Barraco, Phys. Rev. D70 (2004) 043505.

[12] R. Ferraro and F. Fiorini, Phys. Rev. D75 (2007) 084031.

[13] A. Einstein, Sitzungsber. Preuss. Akad. Wiss. (1928) 217; A. Einstein, Math. Annal. 102 (1930) 685. English version in http://www.lrz-muenchen.de/ aunzicker/ae1930.html; F. Gronwald and F.W. Hehl, On the gauge aspects of gravity. In Proc. of the 14th International School on Cosmology and Gravitation, eds. P.G. Bergmann et al. (World Scientific, Singapore, 1996), pp. 148-198.
[14] K. Hayashi and T. Shirafuji, Phys. Rev. D19 (1979) 3524.

[15] H.I. Arcos and J.G. Pereira, Int. J. Mod. Phys. D13 (2004) 2193.

[16] R. Weitzenböck, Invarianten Theorie, (Nordhoff, Groningen, 1923).

[17] J.W. Maluf, J. Math. Phys. 35 (1994) 335.

[18] M. Born and L. Infeld, Proc. R. Soc. A144 (1934) 425; ibid., 147 (1934) 522; ibid., 150 (1935) 141.

[19] A.A. Tseytlin, Nuc. Phys. B501 (1997) 41.

[20] J.P. Gauntlett, J.Gomis and P.K. Townsend, JHEP 9801 (1998) 003.

[21] S. Deser and G.W. Gibbons, Class. Quant. Grav. 15 (1998) 35.

[22] J. A. Feingenbaum, Phys. Rev. D58 (1998) 124023.

[23] J. A. Feingenbaum, P.O. Freund and M. Pigli, Phys. Rev. D57 (1998) 4738.

[24] D. Comelli, Phys. Rev. D72 (2005) 064018.

[25] D. Comelli and A. Dolgov, JHEP 0411 (2004) 062.

[26] J. A. Nieto, Phys. Rev. D70 (2004) 044042.

[27] D. N. Vollick, Phys. Rev. D72 (2005) 084026.

[28] M. Bañados, Eddington-Born-Infeld action for dark energy and dark matter [arXiv:hep-th/0801.4103].

[29] M. Bañados, C. Teitelboim and J. Zanelli, Phys. Rev. Lett. 69 (1992) 1849.

[30] M. Bañados, M. Henneaux, C. Teitelboim and J. Zanelli, Phys. Rev. D48 (1993) 1506. 
[31] S. Carneiro, J. Phys. A40 (2007) 6841.

[32] R. Ferraro, F. Fiorini and G. Giribet, work in progress.

[33] In Ref. 25], the authors have considered exact solutions in two dimensions under the assumption of maximally symmetric spacetimes, while in 22] numerical black holes solutions in four dimensions were explored. 